\title{
ENTWICKLUNG UND VERBESSERUNG WEICHE FÄHIGKEITEN IN DER MODERNEN BILDUNGSUMGEBUNG IM ZUSAMMENHANG MIT EINEM KOMPETENZANSATZ
}

\section{Barabash O. O.}

\section{EINFÜHRUNG}

Das Gesetz der Ukraine „Über Bildung besagt“: Bildung ist die Grundlage für die intellektuelle, spirituelle, physische und kulturelle Entwicklung des Einzelnen, seine erfolgreiche Sozialisierung, sein wirtschaftliches Wohlergehen, der Schlüssel zur Entwicklung einer Gesellschaft, die durch gemeinsame Werte und Kultur und den Staat vereint ist ${ }^{1}$ „Lernen ist ein wesentlicher Bestandteil des Bildungsprozesses, durch den Bildungseinrichtungen den Zweck erreichen wollen, für den sie geschaffen wurden, einschließlich der Entwicklung und Gestaltung des Charakters und der geistigen Fähigkeiten ihrer Schüler sowie ihrer persönlichen Unabhängigkeit" - heißt es in einem Urteil des Europäischen Gerichtshofs. zu den Menschenrechten ${ }^{2}$.

Solche europäischen Standards spielen eine Schlüsselrolle bei der Umsetzung der vorrangigen Aufgabe der modernen ukrainischen Gesellschaft im Bereich der Reform des Bildungssystems - dem Übergang zu innovativem Lernen. Im modernen innovativen Bildungsprozess sind Wissen, Fähigkeiten und Fertigkeiten jedoch nicht an sich wichtig, sondern als Mittel, um ein aussagekräftigeres Ziel zu erreichen - die Bildung einer aktiven, kreativen Person, die dieses Wissen in der Praxis anwenden kann. Dieser Trend zeigt sich insbesondere in der juristischen Ausbildung, wo die Aufgabe der Ausbildung eines praktischen Anwalts auf der Tagesordnung steht.

Separate Forschungen befassen sich mit der Frage, wie die Bedeutung von «Soft Skills" bei der Vorbereitung von Absolventen der Rechtswissenschaften erhöht werden kann. Trotzdem nimmt die Dringlichkeit des Problems der Bildung von „Soft Skills“ künftiger Anwälte im Rahmen ihrer Berufsausbildung nicht ab, sondern wird relevanter, da „Soft Skills“ einer der grundlegenden Mechanismen zur Anpassung eines Anwalts an sozioökonomische Veränderungen ist. So haben die Realitäten des modernen politischen, wirtschaftlichen und sozialen Lebens der Gesellschaft einen Paradigmenwechsel in der Hochschulbildung bestimmt. Das Qualifikationsmodell eines Spezialisten ist eng an das Fach und den Gegenstand der Arbeit gebunden und wurde durch das Kompetenzmodell erheblich ersetzt. Im Kompetenzmodell der Fachausbildung ist der Zweck der Ausbildung nicht nur mit

\footnotetext{
${ }^{1}$ Про освіту : Закон України від 05 вересня 2017 року. Офіиійний вісник Украӥни. 2017. № 78. С. 7.

${ }^{2}$ European Court of Human Rights' Decision "Hasan and Eylem Zenginv. Turkey" from 09.10.2007, case1448/04. § 55. URL : http://hudoc.echr.coe.int/eng?i=001-82580.
} 
den Objekten und Arbeitsobjekten verbunden, mit der Erfüllung bestimmter Funktionen, sondern auch mit interdisziplinären integrierten Anforderungen an das Ergebnis des Bildungsprozesses. Der Erwerb von Kompetenz ermöglicht es, verschiedene unvorhergesehene Situationen zu überwinden, die im Verlauf der beruflichen Tätigkeit auftreten, und zu lernen, in einer Gruppe zu arbeiten. Kompetenz ist eine neue Art der Definition des Zwecks des Bildungsprozesses und markiert den Übergang von überwiegend akademischen Bewertungsstandards zu externer Bewertung der beruflichen und sozialen Bereitschaft von Hochschulabsolventen sowie in engem Zusammenhang mit den Anforderungen des Arbeitsmarktes. Der Besitz von Kompetenzen beinhaltet den Einsatz in einer Reihe von Berufen, wodurch der Umfang ihrer Beschäftigung erweitert werden kann. Es sollte betont werden, dass Kompetenz nicht gleichzeitig der beruflichen Qualifikation entgegensteht und nicht damit identifiziert werden kann. Wir glauben jedoch, dass der Fokus ausschließlich auf dem Kompetenzmodell liegt, das eine erfolgreichere Beschäftigung und eine hohe Produktionsleistung löst, was zu einer geringeren Produktion führt, da enge Spezialisten nicht in der Lage sind, praktische Probleme zu lösen, um die wissenschaftliche und technologische Entwicklung sicherzustellen. Das optimale Modell eines Spezialisten sollte systemischer Natur sein und die Vorteile von Qualifikationsund Kompetenzmodellen berücksichtigen.

Vor diesem Hintergrund muss ein Jurastudent heute über eine Reihe persönlicher Qualitäten, Fähigkeiten und Fertigkeiten verfügen, die einerseits die fortschreitende Entwicklung des Einzelnen und dann der Gesellschaft in einem sich ständig verändernden Umfeld und andererseits die Entwicklung fördern, seine Wettbewerbsfähigkeit erhöhen. Gleichzeitig ist es unter Berücksichtigung der Beschäftigungssituation notwendig zu verstehen, was Arbeitgeber von ihren Arbeitnehmern erwarten. Dies wiederum ermöglicht es den Studenten, während ihres Studiums die erforderlichen Fähigkeiten zu erwerben, um auf dem Arbeitsmarkt wettbewerbsfähig zu werden.

\section{Weiche Fähigkeiten, als persönliche Qualitäten, Fähigkeiten und Fähigkeiten in der Bildungsumgebung}

Die Welterfahrung bestätigt die Machbarkeit der Bildung von «flexiblen» (Soft Skills) und „harten“ (Hard Skills) Fähigkeiten in einem einzigen Bildungsumfeld. Die Bedeutung sozialer Kompetenzen spiegelt sich in den Aktivitäten vieler internationaler Organisationen wider, die Lehrpläne für Juristen akkreditieren und die beruflichen Qualifikationen ihrer Absolventen bescheinigen.

Der moderne Arbeitsmarkt spiegelt einen spürbaren Trend wider, der ein erhebliches Interesse der Arbeitgeber an den „Soft Skills“ von Arbeitssuchenden zeigt. Die meisten Arbeitgeber halten sie für ebenso wichtig wie berufliches 
Wissen und Können. Berufliche Fähigkeiten und Fertigkeiten sind veraltet, und „Soft Skills“ sind immer relevant. In der Ukraine ist das Konzept der „Soft Skills“ relativ neu und unerforscht. Als Reaktion auf die Anforderungen des Arbeitsmarktes bieten verschiedene Ausbildungsagenturen eine Vielzahl von Programmen an, die auf die Entwicklung von „Soft Skills“ abzielen” ${ }^{3}$.

Die Forscher unterscheiden drei Gruppen von Berufen, die unterschiedliche Prioritätsverhältnisse der untersuchten Kompetenzen aufweisen: Berufe, in denen hart über weich herrscht: zum Beispiel Kernphysiker, Designer usw.; Berufe, in denen beide Arten von Fähigkeiten gleichermaßen erforderlich sind: zum Beispiel Anwälte, Buchhalter, Zollbeamte; Berufe, in denen "Soft Skills“" vorherrschen: zum Beispiel Wirtschaft, Politik oder kreative Berufe.

Da diese Begriffe für die Gesellschaft von erheblichem Interesse sind, lohnt es sich, die Definition dieser Konzepte zu lesen. Insbesondere werden sie als „Soft Skills“ oder „Flexible Skills“ übersetzt. Diese Begriffe beantworten die Frage „Wie entwickelt man sich?“ der eine oder andere Bereich. Soft Skills ist ein Sammelbegriff, der sich auf viele Verhaltensweisen bezieht, die Menschen bei der Arbeit helfen, einschließlich Teamarbeit sowie erfolgreicher Sozialisierung. Mit Soft Skills können Sie unabhängig von den Besonderheiten der Aktivität und der Richtung, in der die Person arbeitet, erfolgreich sein. Traditionell werden sie in der Psychologie als soziale Fähigkeiten eingestuft: die Fähigkeit zu überzeugen, sich Menschen zu nähern, zu führen, zwischenmenschliche Kommunikation, Verhandlung, Teamarbeit, persönliche Entwicklung, Zeitmanagement, Gelehrsamkeit, Kreativität und mehr ${ }^{4}$.

Mit „Soft Skills“ können Sie die erworbenen juristischen Kenntnisse und Fähigkeiten anwenden und Ziele erreichen. Während der Ausbildung muss der künftige Anwalt sowohl wettbewerbsorientierte Entwicklungen schaffen als auch deren Umsetzung sicherstellen, damit er den modernen Anforderungen des Arbeitsmarktes sowie nationalen und internationalen Standards gerecht werden kann. Insbesondere ein integrierter Indikator für die Qualität des wissenschaftlichen und pädagogischen Potenzials, die Qualität der Bildung und die internationale Anerkennung (wenn die Hochschuleinrichtung in der letzten veröffentlichten Ausgabe des QS World University Rankings und / oder des Times Higher Education World University Rankings und / oder des Academic Ranking of World den 142. Platz belegt Universities - World Top 500 Universities) wurde entwickelt, um Hochschuleinrichtungen hervorzuheben, die weltweit Anerkennung gefunden haben (der beste Indikator im Jahr 2018 -

\footnotetext{
${ }^{3}$ Длугунович H.A. Softskills як необхідна складова підготовки IT-фахівців. Вісник Хмельницького національного університету. 2014. № 6(219). С. 239-242.

${ }^{4}$ Що таке Soft Skills? В чому різниця Soft i Hard Skills? URL : https://www.welldone.org.ua/shho-take-softskills-v-chomu-riznitsya-soft-i-hard-skills/.
} 
sechs ukrainische Hochschuleinrichtungen $)^{5}$. In Anbetracht dessen sollte die Qualität der Ausbildung künftiger Rechtsanwälte nicht nur durch die Bildung der erforderlichen Fachkompetenzen bestimmt werden, sondern auch durch «nicht traditionelle» für die juristische Ausbildung, die sogenannten „sozialen“ oder „weichen“ Fähigkeiten.

Neben „Soft Skills“ gibt es jedoch „Hard Skills“, dh solche beruflichen Kompetenzen, die nicht nur gleichzeitig mit „Soft Skills“ existieren, sondern von entscheidender Bedeutung sind, die miteinander verbunden und voneinander abhängig sein müssen (was leider der Fall ist) Ort) und nur unter dieser Bedingung ein angemessenes $\mathrm{Ma} \beta$ an Lebensqualität bieten, unsere Bedürfnisse erfüllen und ein Gefühl des Vertrauens in die Zukunft.

„Hard Skills“ werden oft übersetzt als „Hard Skills“ sind technische Fähigkeiten im Zusammenhang mit Aktivitäten im Bereich formalisierter Technologien: Büroarbeit, Logistik, Fahren, Programmieren, Blinddruck usw. Das heißt, dies sind direkte berufliche Fähigkeiten, die ziemlich stabil sind, gut zur Überprüfung verfügbar sind, eine bestimmte Dimension haben und in der Liste der Anforderungen enthalten sind, die in den Stellenbeschreibungen aufgeführt sind ${ }^{6}$.

Die Ergebnisse der heimischen Forschung zeigen, dass die "Soft Skills" eines Hochschulabsolventen für seine Beschäftigung und berufliche Anpassung eine immer wichtigere Rolle spielen. Die überwiegende Mehrheit der Autoren sieht die Bildung von „Soft Skills“ in der aktiven Teilnahme von Studenten in verschiedenen Kreisen, Gewerkschaften, öffentlichen Vereinigungen, wissenschaftlichen Gesellschaften, Studentenregierungen und mehr. Solche Aktivitäten tragen dazu bei, die Kommunikationsfähigkeiten zu verbessern und Verantwortung für Entscheidungen zu übernehmen ${ }^{7}$.

Andere sind sich einig, dass „die Grundlage für die Ausbildung nichttechnischer Fähigkeiten von Studenten industrielle Praxis und Praktika sein können “8. Ein Teil der wissenschaftlichen Gemeinschaft ist im Allgemeinen der Ansicht, dass ,die Entwicklung von Soft Skills keine separate Arbeitsform erfordert und eine Überprüfung bestehender Praktiken erfordert, die durch aktive

\footnotetext{
5 Дубицький Д.П. Якість освіти як фактор впливу на державне фінансування закладу вищої освіти. Формування сучасної моделі управління та підвищення якості менеджменту в системі вищої освіти : матеріали Міжнародної науковопрактичної конференції. Дніпро : Університет митної справи та фінансів, 2018. C. 141-142.

${ }^{6}$ Що таке Soft Skills? В чому різниця Soft i Hard Skills? URL : https://www.welldone.org.ua/shho-take-softskills-v-chomu-riznitsya-soft-i-hard-skills/.

${ }^{7}$ Федорова О.В. Формирование hard skills, soft skills и digital skills у студентов факультета информационных технологий УВО «Университет управления «ТИСБИ» Научно информационный журнал Вестник «ТИСБИ». 2018. № 2. С. 335-341.

${ }^{8}$ Taylor E. Investigating the Perception of Stakeholders on Soft Skills Development of Students: Evidence from South Africa/ Interdisciplinary Journal of e-Skills and Lifelong Learning. 2016. Vol. 12. P. 1-18. doi: http://doi.org/10.28945/3412.
} 
Dialoge mit Studenten, die Berücksichtigung realer Beispiele, Präsentationen und Diskussionen bereichert werden" $"$.

Die Anforderungen der Arbeitgeber an Hochschulabsolventen haben sich heute erheblich geändert. Sie schätzten vor allem die sogenannten ,flexiblen Fähigkeiten“: intellektuelle Fähigkeiten potenzieller Mitarbeiter (Lernfähigkeit, Kreativität, Fähigkeit zum systematischen Denken, kritischer Verstand), persönliche Qualitäten (Stress, Flexibilität, Initiative, Verantwortung) und kommunikativ (Fähigkeit, im Team zu arbeiten, zu verhandeln, Konfliktsituationen zu lösen usw.).

Laut O. Gura lohnt es sich daher, sich auf die folgenden Eigenschaften zu konzentrieren, die im modernen Geschäft Priorität haben:

1. Teamwork - die Fähigkeit, in einem Team zu arbeiten und ihre Aufgaben auszuführen, wobei individuelle Fähigkeiten mit anderen Teammitgliedern kombiniert werden ein gemeinsames Ziel erreichen.

2. Suche und Analyse von Informationen - die Fähigkeit von Spezialisten, Informationen aus verschiedenen Quellen $\mathrm{zu}$ finden, $\mathrm{zu}$ verarbeiten und $\mathrm{zu}$ synthetisieren.

3. Kreativität. Spezialisten suchen ständig nach neuen Lösungen, um potenziellen technologischen Anforderungen und Problemen gerecht zu werden.

4. Entscheidungsfindung - die Fähigkeit, den besten Weg zu finden, um maximale Effizienz und Erfolg des Ergebnisses bei minimalen Kosten für persönliche oder Teamressourcen sicherzustellen.

5. Konfliktmanagement - die Fähigkeit zu verhandeln, Konfliktsituationen zu minimieren und eine angespannte Atmosphäre im Team zu verhindern.

6. Kritisches Denken - die Fähigkeit einer Person, die von außen kommenden Informationen in Frage zu stellen und die Möglichkeit eines eigenen Fehlers anzunehmen.

7. Präsentation - die Fähigkeit, ihre Ideen klar zu artikulieren und anderen in der günstigsten Form zu präsentieren.

8. Führung - die Fähigkeit, Geschäftsentscheidungen $\mathrm{zu}$ treffen und Untergebene zu verwalten.

9. Kommunikationsfähigkeiten - die Fähigkeit, Informationen klar, einfach, effektiv und klar an Menschen weiterzugeben. Eine gut etablierte Kommunikation wird zur Grundlage vieler moderner Entwicklungsmethoden.

10. Flexibilität - die Fähigkeit, negative und unerwartete Änderungen vorzunehmen und sofort nach neuen kreativen Lösungen zu suchen.

\footnotetext{
${ }^{9}$ Раицкая Л., Тихонова Е. Soft skills в представлении преподавателей и студентов российских университетов в кон-тексте мирового опыта. Вестник РУДН. Серия: психология и педагогика. 2018. Т. 3 , № 3. C. 350-363. doi: http://doi.org/10.22363/2313-1683-2018-15-3-350-363.
} 
11. Zeitmanagement - eine Reihe von Techniken und Technologien, mit denen eine Person ihre persönliche Zeit planen kann, um die Effizienz und Rationalität ihrer Verwendung zu erhöhen ${ }^{10}$.

$\mathrm{Zu}$ den vorrangigen Fähigkeiten zählen auch die Fähigkeit zu lernen, Teamwork und Disziplin, die Gewohnheit der Priorisierung sowie die Konzentration auf Ergebnisse. In China ist es beispielsweise nicht üblich, NEIN zu sagen. Sie werden von Kindheit an durch verschiedene Methoden gelehrt, taktvoll «abzulehnen». Wenn ein Chinese Sie ablehnt, sind Sie daher immer zufrieden, als ob Ihnen tatsächlich JA gesagt worden wäre ${ }^{11}$.

Es ist interessant festzustellen, dass die Ergebnisse der Forschung an der Harvard University am Stanford Research Institute zeigen, dass der berufliche Erfolg aufgrund von „harten Fähigkeiten“ nur 15\% beträgt, während „weiche Fähigkeiten" bei $85 \%$ liegen. ${ }^{12}$ Laut Forschern widmet die überwiegende Mehrheit der freien wirtschaftlichen Bildung der Entwicklung der „Soft Skills“ der Schüler nicht die gebührende Aufmerksamkeit. Die Lehrpläne sind mit Disziplinen überladen, die die „harten Fähigkeiten“ der Schüler entwickeln, dh „harte“ Fähigkeiten, die mit dem Wissen über grundlegende und spezielle Disziplinen verbunden sind, praktische Ausbildung erhalten, etc. Wie ein zukünftiger Absolvent in einem Team mit Mitarbeitern, Kunden und Partnern interagiert, wird nicht gelehrt, obwohl diese Fähigkeiten einen starken Einfluss auf die Leistung der Mitarbeiter haben. Dies ist das sogenannte „weiche“ Wissen, das schwer zu verbalisieren ist, Fähigkeiten und Erfahrungen. ${ }^{13}$ So wird die Qualität der Ausbildung von Juristen und ihre Wettbewerbsfähigkeit heute nicht nur durch den Grad der Beherrschung des Fachwissens, der Fähigkeiten und Fertigkeiten (Hard Skills), sondern auch durch die sogenannten flexiblen Fähigkeiten (Soft Skills) anerkannt. Das heißt, die direkte Aufgabe der Universität besteht darin, Bedingungen und Möglichkeiten für die Entwicklung dieser Fähigkeiten auf der Grundlage der Überwachung der Anforderungen der Arbeitgeber bereitzustellen ${ }^{14}$.

$\mathrm{Zu}$ den effektivsten Instrumenten für die Entwicklung von „Soft Skills“ gehören interaktive Lehrmethoden. Dazu gehören: Gruppendiskussionen, Fallstudien, Rollen- und Geschäftsspiele, Debatten. Diese Lehrmethoden

\footnotetext{
${ }^{10}$ Гура О.О. С. Особливості розвитку м’яких навичок студентів IT-спеціальностей. Journal «ScienceRise: Pedagogical Education. 2019. № 4(31). C. 9-10.

${ }^{11}$ Що таке Soft Skills? В чому різниця Soft i Hard Skills? URL : https://www.welldone.org.ua/shho-take-softskills-v-chomu-riznitsya-soft-i-hard-skills/.

12 Янковська О. Звіт за результатами І Національного Форуму «Бізнес і університети». Центр «Розвиток КСВ». К., 27 листопада 2013 p. URL : http://csrukraine.org/wp-content/uploads/2014/04/ForumReport_ Final.pdf.

${ }^{13}$ Коваль К.О. Розвиток "Soft Skills“ у студентів - один з важливих чинників працевлаштування. Вісник Вінницького політехнічного інституту. 2015. № 2. С. 163.

${ }^{14}$ Peggy Klaus. The Hard Truth About Soft Skills. Harper Collins Publishers, 2007. 191 p.
} 
ermöglichen es Ihnen, „Soft Skills“ zu entwickeln, indem Sie in der Praxis bestimmte praktische Situationen simulieren, denen Fachleute bei echten beruflichen Aktivitäten begegnen können.

Ein wichtiger Platz bei der Entwicklung von „Soft Skills“ von Studenten sind Schulungen zu Motivation, Führung, Management, Teamarbeit, Zeitmanagement, Präsentationen und persönlicher Entwicklung, die von Universitäten organisiert werden.

Erwähnenswert ist auch ein solches Instrument zur Entwicklung von «Soft Skills" wie Praktika bei Unternehmen, Institutionen und Organisationen. Praktika wirken sich erheblich auf die Vorbereitung der Studierenden auf den zukünftigen Beruf eines Anwalts im Allgemeinen aus: Entwickeln Sie nicht nur berufliche Fähigkeiten, sondern auch Kommunikationsfähigkeiten. Im Rahmen von Praktika werden Berufsethik gebildet und die netzwerkprofessionellen Kontakte ausgebaut. Meist ist der erfolgreiche Abschluss von Praktika durch Studierende ein entscheidender Faktor bei der Einstellung.

Eine sehr effektive Ressource für die Entwicklung von „Soft Skills“ von Studenten (und gleichzeitig unbrauchbar) - Freiwilligenarbeit. Die aktive Teilnahme an der Freiwilligentätigkeit bildet heute nicht nur eine aktive bürgerliche Position und Werte des Humanismus, sondern trägt auch zur beruflichen Selbstbestimmung bei und erhöht die Motivation, die Disziplinen eines bestimmten Bildungsprogramms zu beherrschen.

Die folgenden Vorteile der Freiwilligentätigkeit bei der Sicherstellung des persönlichen und beruflichen Wachstums von Studenten können unterschieden werden: organische Bildung universeller beruflicher Kompetenzen; Erfahrung in sozial orientierten Aktivitäten sammeln, bürgerliche Qualitäten entwickeln; Erhalten der notwendigen Kontakte, Verbindungen; Erhöhung der Verantwortung und Unabhängigkeit. Es sollte jedoch anerkannt werden, dass in modernen Bildungsaktivitäten das Potenzial der Freiwilligenarbeit praktisch nicht als Instrument zur persönlichen und beruflichen Weiterentwicklung, zur Entwicklung universeller und beruflicher Kompetenzen von Studenten genutzt wird. Um das Potenzial der Freiwilligenarbeit an Universitäten auszuschöpfen, müssen daher bestimmte Bedingungen geschaffen werden, nämlich: Unterstützung von Freiwilligeninitiativen für Studenten durch die Universität; Rückmeldung (Verstärkung) der Ergebnisse des persönlichen beruflichen Wachstums, die der Student im Rahmen der Freiwilligenarbeit erzielt hat; gemeinsame Teilnahme von Schülern und Lehrern an Freiwilligenarbeit usw.

Es ist jedoch anzumerken, dass das Prinzip der Vernetzung und Untrennbarkeit von „harten“ und „weichen“ Kompetenzen, die die Grundlage für die Berufsausbildung bilden, wichtig ist. Daher werden emotionale und kommunikative Qualitäten in der ausländischen Bildungspraxis nicht umsonst als 
„Soft Skills“ oder „Soft Skills“ betrachtet, die „Hard Skills» ergänzen technische / harte / berufliche Fähigkeiten. Im Gegensatz zu letzteren, die normalerweise gut messbar, ziemlich stabil, mit bestimmten Designs identisch sowie Teil von Stellenbeschreibungen und Qualifikationen sind, sind „Soft Skills“ universell und wichtig für eine erfolgreiche berufliche und lebensbezogene Selbstbestimmung jeder Person, unabhängig vom Beruf. Dazu gehören die Fähigkeit zu kommunizieren, Führung, Zusammenarbeit, Diplomatie, Aufbau von Beziehungen; Team-, Öffentlichkeits-, Denk -Fähigkeiten; Fähigkeit, ihre Ideen zu präsentieren, offene Aufgaben, einschließlich Sozialplan usw., kreativ zu lösen usw. Diese Kompetenzen in der modernen Welt werden neben beruflichen Kompetenzen als wichtiges Bildungsergebnis angesehen.

Daher sind „harte" und „weiche“ Kompetenzen trotz der unterschiedlichen Interpretationen der Definition und der Aspekte der Untersuchung des Problems teilweise miteinander verbunden und voneinander abhängig. „Weiche“ Kompetenzen beziehen sich auf Kommunikationsfähigkeiten, die eine notwendige Voraussetzung für eine erfolgreiche Arbeit in beruflichen Aktivitäten sind. «Harte» Kompetenzen sind die Fähigkeit, bestimmte berufliche Aufgaben auszuführen. Die ständige Weiterentwicklung dieser beiden Arten von Kompetenzen bestimmt die erfolgreiche Erreichung ihrer persönlichen und beruflichen Ziele und erhöht ihre Wettbewerbsfähigkeit auf dem Arbeitsmarkt.

\section{Die rolle der Bildungsumgebung bei der Bildung weicher Fähigkeiten}

Umfragen unter Arbeitgebern zufolge konzentriert sich die heutige inländische Hochschulbildung leider nicht auf das Konzept der „Soft Skills“ als Hauptanforderung des Arbeitsmarktes für Hochschulabsolventen. Die meisten Lehrer und Schüler haben keine Ahnung von dem untersuchten Phänomen, da die Sozial- und Geisteswissenschaften wie Fremdsprachen und Management die einzige Quelle für nichttechnische Fähigkeiten künftiger Fachkräfte sind. Gleichzeitig spielt das Bildungsumfeld selbst eine besondere Rolle bei der Bildung von „Soft Skills“'15. Ein solches Umfeld sollte den Wunsch der Schüler nach Entwicklung fördern und neue Erfahrungen, berufliches Wachstum und Feedback von Lehrern und Arbeitgebern sammeln. Die aktive Teilnahme an einem der ausgewählten Bereiche gibt den Studierenden die Möglichkeit, ihr eigenes Potenzial einzuschätzen und zu verstehen, welche Kompetenzen überhaupt entwickelt werden müssen. Die Universitäten bewegen sich wirklich (wenn auch ungleichmäßig) von einem translationalen Modell (Vorlesungsseminar) zu einem integrierten Modell, das Design, Fallstudien und Praktika umfasst, bei denen Simulatoren (Modelle, Modelle usw.) verwendet

\footnotetext{
${ }^{15}$ Гура О.О. Особливості розвитку м’яких навичок студентів IT-спеціальностей. Journal «ScienceRise: Pedagogical Education. 2019. № 4(31). C. 8.
} 
werden. Der Besitz solcher Fähigkeiten bestimmt maßgeblich den Erfolg zukünftiger beruflicher Aktivitäten des Studenten und trägt zur erfolgreichen Umsetzung in einer sich schnell verändernden Welt bei.

Die Erfahrung zeigt, dass es zwei Möglichkeiten gibt, „flexible“ Fähigkeiten zu entwickeln: spezielle Kurse innerhalb des variablen Teils des Lehrplans einzuführen, ebenso wie ausländische Universitäten (Harvard University, Stanford Research Institute usw.), oder sich innerhalb von Disziplinen zu bilden, die mit außerschulischen Aktivitäten vereinigt sind. Arbeit, die in der Praxis der inländischen Universitäten üblicher ist.

Die Analyse der Auslandserfahrung in der Erforschung von Problemen bei der Bildung von «Hard Skills» und „Soft Skills“ von Studierenden juristischer Fachrichtungen lässt jedoch folgende Schlussfolgerungen zu:

1. Bei der Ausbildung künftiger Fachkräfte müssen nicht nur die Anforderungen der geltenden Rechtsvorschriften zu Bildung und beruflichen Standards berücksichtigt werden, sondern auch die Interessengruppen und andere Teilnehmer am Bildungsprozess.

2. Es ist notwendig, den Prozess der Bildung der betrachteten Fähigkeiten bei Studierenden juristischer Fachrichtungen eingehender zu untersuchen.

3. Aufgrund des Mangels an methodischem Material muss ein Diagnosesystem entwickelt werden, mit dem die Beherrschung der „Soft Skills“ durch die Schüler beurteilt werden kann.

Unter dem Gesichtspunkt der Sicherstellung der Wettbewerbsfähigkeit eines Absolventen einer inländischen Universität auf dem modernen Arbeitsmarkt ist es ein kompetenter Ansatz in der Bildung, der es Ihnen ermöglicht, die Persönlichkeit eines Fachmanns zu formen, der bereit ist, sich der internationalen Berufsgemeinschaft anzuschließen und effektiv berufliche Tätigkeiten auszuüben. Es ist kein Zufall, dass der Begriff „Soft Skills“ (universelle Kompetenzen, einheitliche Fähigkeiten) bei der Entwicklung von Schulungsprogrammen, Auffrischungskursen und Programmen zur beruflichen Weiterentwicklung führend geworden ist ${ }^{16}$.

Kompetenz kann definiert werden als ein soziales Arbeitsmerkmal des Wissens, der Fähigkeiten, Fertigkeiten und beruflich wichtiger Qualitäten und Motivationsmerkmale des zukünftigen Anwalts, der über die für eine erfolgreiche Arbeitserfüllung erforderlichen Voraussetzungen und die relevanten Anforderungen der Position verfügt. Kompetenz - ein Merkmal potenzieller Qualität, das die meisten Elemente der Bereitschaft eines Anwalts für produktive und effektivste Tätigkeiten bestimmt. Kompetenzen modellieren das Verhalten eines idealen Anwalts als Spezialist, der eine bestimmte Art von beruflicher

\footnotetext{
${ }^{16}$ Mintzberg, H. Managers Not MBAs: A Hard Look at the Soft Practice of Managing and Management Development. Berrett-Coehler Publishers, 2004. 464 p.
} 
Tätigkeit ausübt. Um das konstruierte Idealbild in der objektiven Realität zu verwirklichen, muss sich eine Person der Prioritätsmerkmale ihrer Tätigkeit bewusst sein, die bestimmte Anstrengungen erfordern und das entsprechende Ergebnis sicherstellen.

Im Gegensatz zu Kompetenz sind sich die Forscher bei der Betrachtung von Kompetenz einig, dass Kompetenz ein integraler, dh umfassenderer Begriff ist. ${ }^{17}$ Dieses Konzept beinhaltet die Anwesenheit eines zukünftigen Anwalts mit einer bestimmten Anzahl von Kompetenzen. Jede der Kompetenzen hat den notwendigen Entwicklungsstand, ist ein Merkmal der Tiefe des Wissens des Mitarbeiters seines Unternehmens, seines Verständnisses des Wesens der Arbeit sowie der Mittel und Wege, um Ziele zu erreichen. Kompetenz wird durch die Anhäufung von Berufserfahrung bestimmt. Daher ist es unserer Meinung nach zweckmäßig, die Kompetenz als Hauptkategorie (Grund-) des Kompetenzansatzes in einem Anwaltsberuf zuzuweisen und durch ihn eine Berufsausbildung zu erwerben. In diesem Fall fungiert die Kompetenz als sekundäre (abgeleitete) Kategorie.

Manifestation der Wettbewerbsfähigkeit als wirksamer Mechanismus der sozialen Anpassung an Veränderungen, Bildung der Notwendigkeit einer kontinuierlichen Entwicklung beruflicher Kompetenzen, die für die erfolgreiche soziale und berufliche Selbstverwirklichung und Erreichung von Zielen erforderlich sind, Einsatz von Fähigkeiten, Kenntnissen und Fertigkeiten in beruflichen Tätigkeiten aufgrund rascher Veränderungen in den Bereichen Soziales, Wissenschaft und Technik Wirtschaftsleben der Gesellschaft. Vor diesem Hintergrund ist die Verbindung zwischen ,harten“ und ,weichen“Kompetenzen wichtig.

Experten des Weltwirtschaftsforums haben eine Prognose erstellt, in der sie zehn Schlüsselkompetenzen identifiziert haben, die 2020 gefragt sein werden. Insbesondere wird laut Prognose die Fähigkeit, komplexe Probleme zu lösen, die wichtigste Kompetenz sein. Die zweitwichtigste Kompetenz ist kritisches Denken und die dritte ist Kreativität. Auf diese Kompetenzen folgen Personalmanagementkompetenzen, Koordinations- und Interaktionsfähigkeiten, emotionale Intelligenz, Urteilsvermögen und Entscheidungsfindung, Kundenorientierung, Verhandlungsfähigkeiten und kognitive Flexibilität ${ }^{18}$. Beachten Sie, dass alle oben genannten Kompetenzen zu den «Soft Skills“" gehören.

Wie bereits erwähnt, können „Soft Skills“ als eine Kombination aus bestimmten persönlichen Qualitäten, emotionaler Intelligenz und Kommunikationskompetenz definiert werden, die es einem Spezialisten ermöglichen, beruflichen Erfolg zu erzielen.

\footnotetext{
${ }^{17}$ Mintzberg, H. Managers Not MBAs: A Hard Look at the Soft Practice of Managing and Management Development. Berrett-Coehler Publishers, 2004. 464 p.

${ }^{18}$ Ананьева, Т. Десять компетенций, которые будут востребованы в 2020 году. URL : http://www.tananyeva.com/ single-post/ (дата звернення: 25.05.2020).
} 
Wenn wir die Betrachtung weicher Kompetenzen als eine Reihe nicht spezialisierter überprofessioneller Fähigkeiten betrachten, die eine erfolgreiche Teilnahme am Arbeitsprozess und eine hohe Produktivität bestimmen, können wir die wichtigsten Faktoren identifizieren, die die Wirksamkeit ihrer Bildung im Bildungsumfeld bestimmen: Unmittelbarkeit (Technologie), Kontext ihre Anwendung, intrinsische Motivation der Person. Es gibt verschiedene Möglichkeiten, um weiche Kompetenzen zu entwickeln: Schulungen, Schulungsprogramme für Unternehmen, Mentoring und Coaching.

Die Wirksamkeit der Entwicklung und Ausbildung von Soft-Kompetenzen ist angesichts der humanitären Komponente, die keinen klar definierten Kriterien und einer eindeutigen Bewertung unterliegt, schwer zu überwachen. Vor diesem Hintergrund sind mit der Entwicklung weicher Kompetenzen bestimmte Risiken verbunden: Nichtverbesserung von Kompetenzen, das Risiko, Geld für die Ausbildung und Entwicklung von Kompetenzen zu verlieren, emotionaler Stress, das Risiko der Unzufriedenheit mit ihren Kompetenzen ${ }^{19}$. Die identifizierten Risiken können jedoch durch vorherige Analyse des Entwicklungsgrades jeder der erforderlichen Kompetenzen sowie durch die richtige Motivation und die Festlegung klarer Ziele und Vorgaben minimiert werden.

Das Wissen und Verstehen über berufliche Aufgaben und die Anwendung von Kompetenzen sind die Grundvoraussetzung für die Schaffung von Konsistenz, Kohärenz und Integrität bei der Vermittlung „weicher“ Kompetenzen. Die logische Begründung und das rationale Bewusstsein des Arbeitnehmers für die Aufgaben ermöglichen es, die am meisten nachgefragten Fähigkeiten $\mathrm{zu}$ bestimmen und im Einklang mit den Interessen des Arbeitgebers zu bestimmen, welche Fähigkeiten von Fachleuten benötigt werden. Für das Funktionieren von Fähigkeiten in realen Situationen ist es notwendig, den organisatorischen und beruflichen Kontext zu reproduzieren, die Merkmale der beruflichen Tätigkeit darzustellen und $\mathrm{zu}$ verstehen sowie berufliche Probleme $\mathrm{zu}$ identifizieren, $\mathrm{zu}$ formulieren und $\mathrm{zu}$ lösen. Die Bildung solcher Fähigkeiten zielt darauf $a b$, die Fähigkeit zu entwickeln, die Variabilität der Situation zu sehen und zu unterscheiden. Dementsprechend ist ersichtlich, dass der Einsatz von „Soft Skills" nur möglich ist, wenn die Bedingungen für die Fähigkeit, jeden Tag unterschiedliche Verhaltensweisen in der Belegschaft anzuwenden, organisatorische und persönliche Interessen zu verstehen, Prioritäten zu setzen und die richtige Wahl zu treffen.

Einheitliche Fähigkeiten, dh „weiche“ Kompetenzen, umfassen die konsequente Bearbeitung von Situationen, die den zukünftigen Anwalt dazu ermutigen, unabhängige Entscheidungen zu treffen und eigene Entscheidungen zu treffen, für die er bereit ist, verantwortlich zu sein. Dementsprechend kann der

${ }^{19}$ Уиддет С., Холлифорд С. Руководство по компетенциям. Москва : ГИППО, 2013. С. 68. 
Schluss gezogen werden, dass der Prozess der Verwendung von „Soft Skills“ viel komplexer ist als der Einsatz von „Hard Skills“.

Universelle Kompetenzen sind ein wichtiger Bestandteil des Fachausbildungsmodells und der Fachmodelle, die die Mittel und Ziele der juristischen Hochschulbildung definieren. Diese Art von Kompetenzen im Prozess des Hochschulstudiums wird indirekt in Klassen gebildet, in denen bestimmte Rechtsdisziplinen studiert werden. Wir halten es jedoch für erforderlich, einen Kurs wie „Fremdsprache für Anwälte“ als eine Disziplin zu unterscheiden, die ein erhebliches Potenzial für universelle Kompetenzen aufweist. als die Fähigkeit zu überzeugen, effektiv zwischenmenschliche Kommunikation durchzuführen, in einem Team zu arbeiten, zu verhandeln, den Wunsch nach persönlicher und beruflicher Entwicklung. All diese Fähigkeiten erwirbt der Student im Rahmen der Kommunikation in einer Fremdsprache, wodurch er künftig ein internationaler Anwalt werden kann.

So erfolgt die Einbeziehung der Disziplinen „Fremdsprache für Anwälte" in das Analogon künftiger beruflicher Tätigkeiten, Modellierung in Bildungssituationen ausländischer Kommunikationsprozesse zur Lösung beruflicher Probleme, nämlich Projektaufgaben, Teilnahme an Geschäftsspielen, Reden auf wissenschaftlichen Konferenzen, Verteidigung eines Diploms im Ausland Sprache ermöglichen es den Schülern, sich auf die Zukunft vorzubereiten $^{20}$.

\section{SCHLUSSFOLGERUNG}

Moderne Veränderungen der Art und des Inhalts der Arbeit aufgrund von Globalisierungsprozessen, hohen Raten der wissenschaftlichen und technologischen Entwicklung und Informatisierung der Arbeitskräfte erfordern angemessene Änderungen in der Arbeit mit Mitarbeitern moderner Organisationen und beinhalten den Übergang von der Qualifikation des Rechtsberufs als Merkmal beruflicher Fähigkeiten. spiegelt auch die geschäftlichen und persönlichen Qualitäten eines Anwalt-Spezialisten wider. Es ist die Kompetenz eines Anwalts, die maßgeblich den Erfolg der Anwaltschaft auf dem Arbeitsmarkt bestimmt. Die Haupttrends bei der Verbesserung des Ausbildungssystems zur Steigerung ihrer Wettbewerbsfähigkeit auf dem Arbeitsmarkt beschränken sich daher hauptsächlich auf die Verwendung eines kompetenzbasierten Ansatzes zur Entwicklung von Hochschulstandards, insbesondere: Einführung einer kontinuierlichen Überwachung der Bedürfnisse der Arbeitgeber in bestimmten Kompetenzen; Neuausrichtung des Bildungsinhalts (Bildungsprofis, Arbeitsprogramme der Disziplinen) auf

\footnotetext{
${ }^{20}$ Shaulska L. V., Sereda G. V., Shkurat M. Y. The Development of Soft Skills in the Provision of Competitiveness of Graduates. Економічний вісник Донбасу. 2015. № 4(42). С. 180.
} 
wirksame Indikatoren für die Ausbildung unter Berücksichtigung der gebildeten Kompetenzen; Aufnahme von Ausbildungsdisziplinen in den Lehrplan, die die Entwicklung kognitiver und sozio-emotionaler Fähigkeiten fördern; Entwicklung von Instrumenten zur Überprüfung des Bildungsgrades kognitiver und sozioemotionaler Fähigkeiten (,Soft Skills“).

Die Verwendung eines kompetenzbasierten Ansatzes zur Entwicklung von Hochschulstandards im Zusammenhang mit der Anwendung von «Soft Skills" weist eine Reihe von Besonderheiten auf:

1) konzeptionelle Begründung für die Einbettung des Modells in ein modernes Lernsystem (es geht eher um die Optimierung der Kombination traditioneller und innovativer Lernansätze als um einen vollständigen Übergang $\mathrm{zu}$ dieser Technologie);

2) integrativer Ansatz, der die Entwicklung von Kursen beinhaltet, die das Panorama-Denken formen und Fähigkeiten zu Metasubjekten entwickeln;

3) komplexe wissenschaftliche, methodische, technologische, informative Unterstützung und Unterstützung der Lehrertätigkeit (Erstellung von Videos, Algorithmus zur Gestaltung der technologischen Karte der Vorlesung oder des Seminars, elektronisches System zur Überwachung der akademischen Leistungen der Schüler);

4) Teamarbeit: Hochschullehrer, Studenten;

5) Mobilität und einfacher Zugang zu Bildungsressourcen.

Darüber hinaus ist es bei der Entwicklung von Technologien und Formen der Entwicklung von „Soft Skills“ möglich, dem Modell der Umgestaltung des Bildungsprozesses $\mathrm{zu}$ folgen. Zum Beispiel die Einrichtung von Kommunikationslabors, Projektbüros, Forschungsgruppen von Lehrern, die Einführung eines nichtlinearen Stundenplans, die Leitung verschiedener Schulen (zum Beispiel die School of Young Lawyer auf der Grundlage der nach Ivan Franko benannten Lviv National University, die School of Young Lawyers der Kyiv National University nach Taras Shevchenko) usw.

Das Arsenal an Instrumenten zur Entwicklung von „Soft Skills“ von Schülern im modernen Bildungsprozess ist daher recht breit. Dazu gehören: projektbasierte Ausbildung von Studenten in einem Team; breite Einführung interaktiver Lehrmethoden in den Bildungsprozess (Gruppendiskussionen; Fälle; Rollenspiele, Geschäftsspiele; Schulungen; Debatten usw.); Praktika bei Unternehmen, Institutionen und Organisationen; Freiwilligenarbeit; praktische Erfahrung in Studentengruppen; Arbeit in Rechtskliniken, Ausbildung in berufsorientierten Tätigkeiten, einschließlich internationaler Projekte usw.).

Der Schlüssel zum Erfolg bei der Entwicklung von Soft Skills von Studenten und damit zur Steigerung ihrer Wettbewerbsfähigkeit ist die Bildung eines sonderpädagogischen Umfelds der Universität, das es ermöglicht, diese 
Fähigkeiten $\mathrm{ab}$ dem ersten Studienjahr in der Praxis zu verbessern. Die Hauptforschungsaufgabe besteht darin, die erfolgreichen Erfahrungen ausländischer Universitäten bei der Entwicklung „flexibler Fähigkeiten“ von Studenten zu analysieren und zusammenzufassen sowie nach neuen Instrumenten für die Entwicklung „,weicher Fähigkeiten“ zu suchen.

\section{ZUSAMMENFASSUNG}

Der Artikel untersucht die Natur von „Soft Skills“ im modernen Bildungsumfeld im Zusammenhang mit Trends im Interesse der Arbeitgeber an solchen Fähigkeiten bei Arbeitssuchenden. Es wird darauf hingewiesen, dass das Arsenal an Instrumenten zur Entwicklung von „Soft Skills“ von Schülern im modernen Bildungsprozess ziemlich breit ist. Dazu gehören: projektbasierte Ausbildung von Studenten in einem Team; breite Einführung interaktiver Lehrmethoden in den Bildungsprozess (Gruppendiskussionen; Fälle; Rollenspiele, Geschäftsspiele; Schulungen; Debatten usw.); Praktika bei Unternehmen, Institutionen und Organisationen; Freiwilligenarbeit; praktische Erfahrung in Studentengruppen; Arbeit in Rechtskliniken, Ausbildung in berufsorientierten Tätigkeiten, einschließlich internationaler Projekte usw.).

Es wird betont, dass das Qualifikationsmodell eines Spezialisten eng mit dem Gegenstand und Gegenstand der Arbeit verbunden ist und durch das Kompetenzmodell erheblich ersetzt wurde. Im Kompetenzmodell der Fachausbildung ist der Zweck der Ausbildung nicht nur mit den Objekten und Arbeitsobjekten verbunden, mit der Erfüllung bestimmter Funktionen, sondern auch mit interdisziplinären integrierten Anforderungen an das Ergebnis des Bildungsprozesses. Der Erwerb von Kompetenz ermöglicht es, verschiedene unvorhergesehene Situationen zu überwinden, die im Verlauf der beruflichen Tätigkeit auftreten, und zu lernen, in einer Gruppe zu arbeiten. Kompetenz ist eine neue Art der Definition des Zwecks des Bildungsprozesses und markiert den Übergang von überwiegend akademischen Bewertungsstandards zu externer Bewertung der beruflichen und sozialen Bereitschaft von Hochschulabsolventen sowie in engem Zusammenhang mit den Anforderungen des Arbeitsmarktes. Es wird der Schluss gezogen, dass der Schlüssel zum Erfolg bei der Entwicklung von „Soft Skills“ von Studenten und damit zur Steigerung ihrer Wettbewerbsfähigkeit in der Bildung eines sonderpädagogischen Umfelds der Universität liegt, das es ermöglicht, diese Fähigkeiten ab dem ersten Studienjahr in der Praxis zu verbessern. Die Hauptforschungsaufgabe besteht darin, die erfolgreichen Erfahrungen ausländischer Universitäten bei der Entwicklung ,flexibler Fähigkeiten" von Studenten zu analysieren und zusammenzufassen sowie nach neuen Instrumenten für die Entwicklung «weicher Fähigkeiten» zu suchen. 


\section{LITERATURVERZEICHNIS}

1. Ананьева, Т. Десять компетенций, которые будут востребованы в 2020 году. URL : http://www.tananyeva.com/ single-post/ (дата звернення: 25.05.2020).

2. Гура О.О. С. Особливості розвитку м'яких навичок студентів ITспеціальностей. Journal «Science Rise: Pedagogical Education. 2019. № 4(31). C. 8-15. DOI: 10.15587/2519-4984.2019.172007.

3. Длугунович H.A. Softskills як необхідна складова підготовки ІТ-фахівців. Вісник Хмельнищького національного університету. 2014. № 6(219). С. 239-242.

4. Дубицький Д.П. Якість освіти як фактор впливу на державне фінансування закладу вищої освіти. Формування сучасної моделі управління та підвищення якості менеджменту в системі вищої освіти : матеріали Міжнародної науково практичної конференції. Дніпро : Університет митної справи та фінансів, 2018. С. 141-142.

5. Коваль К.О. Розвиток «softskills» у студентів - один 3 важливих чинників працевлаштування. Вісник Віннищького політехнічного інституту. 2015. № 2. C. 162-167.

6. Про освіту : Закон України від 05 вересня 2017 року. Офіційний вісник України. 2017. № 78. С. 7.

7. Уиддет С., Холлифорд С. Руководство по компетенциям. Москва : ГИППО, 2013. $228 \mathrm{c.}$

8. Федорова О.В. Формирование hard skills, soft skills и digital skills у студентов факультета информационных технологий УВО «Университет управления «ТИСБИ». Научно информачионный журнал Вестник «ТИСБИ». 2018. № 2. С. 335-341.

9. Що таке Soft Skills? В чому різниця Soft i Hard Skills? URL : https://www.welldone.org.ua/shho-take-soft-skills-v-chomu-riznitsya-soft-i-hardskills/.

10. Янковська О. Звіт за результатами I Національного Форуму «Бізнес $\mathrm{i}$ університети». Центр «Розвиток КСВ». Київ, 27 листопада2013 p. URL : http://csrukraine.org/wp-content/uploads/2014/04/ForumReport_Final.pdf.

11. European Court of Human Rights' Decision "Hasan and Eylem Zenginv. Turkey" from 09.10.2007, case1448/04. § 55. URL : http://hudoc.echr.coe.int/ eng?i=001-82580.

12. Peggy Klaus. The Hard Truth About Soft Skills. Harper Collins Publishers, 2007. $191 \mathrm{p}$.

13. Shaulska L. V., Sereda G. V., Shkurat M. Y. The Development of Soft Skills in the Provision of Competitiveness of Graduates. Економічний вісник Донбасу. 2015. № 4(42). С. 177-180. 
14. Taylor E. Investigating the Perception of Stakeholders on Soft Skills Development of Students: Evidence from South Africa. Interdisciplinary Journal of e-Skills and Lifelong Learning. 2016. Vol. 12. P. 1-18. DOI: http://doi.org/ $10.28945 / 3412$.

\section{Information about author: Barabash O. O., Doctor of Law,} Associate Professor at the Department of Theory and History of State and Law, Constitutional and International Law Lviv State University of Internal Affairs 26, Gorodotska str., Lviv, 79000, Ukraine 